\title{
The Evolutionary $p(x)$-Laplacian Equation with a Partial Boundary Value Condition
}

\author{
Huashui Zhan $\mathbb{D D}^{1}$ and Zhen Zhou $\mathbb{D D}^{2}$ \\ ${ }^{1}$ School of Applied Mathematics, Xiamen University of Technology, Xiamen 361024, China \\ ${ }^{2}$ School of Science, Jimei University, Xiamen 361021, China \\ Correspondence should be addressed to Huashui Zhan; huashuizhan@163.com
}

Received 15 January 2018; Accepted 6 March 2018; Published 18 April 2018

Academic Editor: Chris Goodrich

Copyright (c) 2018 Huashui Zhan and Zhen Zhou. This is an open access article distributed under the Creative Commons Attribution License, which permits unrestricted use, distribution, and reproduction in any medium, provided the original work is properly cited.

\begin{abstract}
Consider a diffusion convection equation coming from the electrorheological fluids. If the diffusion coefficient of the equation is degenerate on the boundary, generally, we can only impose a partial boundary value condition to ensure the well-posedness of the solutions. Since the equation is nonlinear, the partial boundary value condition cannot be depicted by Fichera function. In this paper, when $\alpha<p^{-}-1$, an explicit formula of the partial boundary on which we should impose the boundary value is firstly depicted. The stability of the solutions, dependent on this partial boundary value condition, is obtained. While $\alpha>p^{+}-1$, the stability of the solutions is obtained without the boundary value condition. At the same time, only if $\alpha>0$ and $p^{-}>1$ can the uniqueness of the solutions be proved without any boundary value condition.
\end{abstract}

\section{Introduction and the Main Results}

The evolutionary $p(x)$-Laplacian equation

$$
u_{t}=\operatorname{div}\left(|\nabla u|^{p(x)-2} \nabla u\right), \quad(x, t) \in Q_{T}=\Omega \times(0, T)
$$

comes from a new interesting kind of fluids, the so-called electrorheological fluids (see $[1,2]$ ), where $\Omega \subset \mathbb{R}^{N}$ is a bounded domain with $C^{2}$ smooth boundary $\partial \Omega$ and $p(x)$ is a measurable function. Equation (1) with the initial value

$$
u(x, 0)=u_{0}(x), \quad x \in \Omega
$$

and the homogeneous boundary value

$$
u(x, t)=0, \quad(x, t) \in \partial \Omega \times(0, T)
$$

has been researched widely; one can refer to [3-6] et al.

$$
\begin{aligned}
& \text { If } p(x)=p \\
& \begin{array}{l}
\frac{\partial u}{\partial t}-\operatorname{div}\left(a(x)|\nabla u|^{p-2} \nabla u\right)-b^{i}(x) D_{i} u+c(x, t) u \\
\quad=f(x, t), \quad(x, t) \in Q_{T}
\end{array}
\end{aligned}
$$

was considered by Yin and Wang [7], where $a(x)$ may be degenerate. Instead of the usual boundary condition (3), they classified the boundary into three parts: the nondegenerate boundary, the weakly degenerate boundary, and the strongly degenerate boundary, by means of a reasonable integral description. The boundary value condition should be supplemented definitely on the nondegenerate boundary and the weakly degenerate boundary. Even earlier, they had studied a simpler equation

$$
u_{t}=\operatorname{div}\left(\rho^{\alpha}|\nabla u|^{p-2} \nabla u\right), \quad(x, t) \in Q_{T},
$$

in [8]. Here $\rho(x)=\operatorname{dist}(x, \partial \Omega), \alpha>0$, and $p>1$. They showed that only if $\alpha<p-1$, the usual boundary value condition (3) can be imposed; while $\alpha \geq p-1$, the uniqueness of the solution can be proved without any boundary value condition.

In this paper, we will consider the evolutionary equation

$$
u_{t}=\operatorname{div}\left(\rho^{\alpha}|\nabla u|^{p(x)-2} \nabla u\right)+g^{i}(x) D_{i}(b(u)) \text {, }
$$


with the initial value (2) and with a partial boundary condition

$$
u(x, t)=0, \quad(x, t) \in \Sigma_{p} \times(0, T)
$$

where $g^{i}(x) \in C^{1}(\bar{\Omega}), D_{i}=\partial / \partial x_{i}, b(s) \in C^{1}(\mathbb{R})$, and

$$
\Sigma_{p}=\left\{x \in \partial \Omega: g^{i}(x) \neq 0\right\}
$$

In the context, $p(x)$ is a $C^{1}(\bar{\Omega})$ function and the definitions of the function spaces with variable exponents $L^{p(x)}(\Omega), W^{1, p(x)}(\Omega), W_{0}^{1, p(x)}(\Omega)$ can be found in [9-16] et al. We denote that

$$
\begin{aligned}
& p^{+}=\max _{x \in \bar{\Omega}} p(x), \\
& p^{-}=\min _{x \in \bar{\Omega}} p(x) .
\end{aligned}
$$

The weak solutions are defined as follows.

Definition 1. If a function $u(x, t)$ satisfies

$$
\begin{aligned}
& u \in L^{\infty}\left(Q_{T}\right), \\
& u_{t} \in L^{2}\left(Q_{T}\right), \\
& \rho^{\alpha}|\nabla u|^{p(x)} \in L^{1}\left(Q_{T}\right), \\
& \iint_{Q_{T}}\left[\frac{\partial u}{\partial t} \varphi+\rho^{\alpha}|\nabla u|^{p(x)-2} \nabla u \cdot \nabla \varphi+b(u) g^{i}(x) \varphi_{x_{i}}\right. \\
& \left.\quad+b(u) g_{x_{i}}^{i} \varphi\right] d x d t=0,
\end{aligned}
$$

for any function $\varphi \in L^{\infty}\left(0, T ; W_{0}^{1, p(x)}(\Omega)\right)$, then we say that $u(x, t)$ is the weak solution of $(6)$. The initial value (2) is true in the sense of

$$
\lim _{t \rightarrow 0} \int_{\Omega}\left|u(x, t)-u_{0}(x)\right| d x=0 .
$$

The partial boundary condition (7) is true in the sense of trace.

If

$$
\begin{array}{r}
u_{0} \in L^{\infty}(\Omega), \\
\rho^{\alpha}\left|\nabla u_{0}\right|^{p^{+}} \in L^{1}(\Omega),
\end{array}
$$

we add some restrictions on $b(s)$, by a similar method to that in $[17,18]$ and the existence of the weak solution of (6) with the initial value (2) can be proved. If $0<\alpha<p^{-}-1$, we can prove that the weak solution $u \in L^{\infty}\left(0, T ; W^{1, \gamma}(\Omega)\right)$ for some $\gamma>1$. Then the existence of the weak solutions of (6) with the initial-boundary value conditions can be obtained. The main aim of this paper is to study the stability of the weak solutions.

Firstly, we mainly pay close attention to the stability of the weak solutions based on the partial boundary value conditions.
Theorem 2. Let $b(s)$ be a Lipschitz function and $u(x, t)$ and $v(x, t)$ be two weak solutions of (6) with the different initial values $u_{0}(x)$ and $v_{0}(x)$, respectively, and with the same partial homogeneous boundary value

$$
\left.u\right|_{\Sigma_{p} \times(0, T)}=0=\left.v\right|_{\Sigma_{p} \times(0, T)} .
$$

If $b(s)$ is a Lipschitz function and

$$
\begin{gathered}
p^{+}>2, \\
p^{-}-1>\alpha \geq \frac{p^{-}-1}{p^{+}-2}
\end{gathered}
$$

then

$$
\begin{array}{r}
\int_{\Omega}|u(x, t)-v(x, t)| d x \leq \int_{\Omega}\left|u_{0}(x)-v_{0}(x)\right| d x, \\
\forall t \in[0, T),
\end{array}
$$

where $\Sigma_{p}$ is the part of the boundary expressed as (8).

Secondly, if $\alpha \geq p^{-}-1$, the weak solutions of (6) cannot be defined as the trace on the boundary and the boundary value condition cannot be used. In order to overcome this difficulty, we will introduce a new kind of the weak solutions in Section 3 and the stability of the weak solutions can be proved when $\alpha>p^{+}-1$.

Theorem 3. Let $u$ and $v$ be two weak solutions of (6) with the different initial values $u(x, 0)$ and $v(x, 0)$, respectively. If $\alpha>$ $p^{+}-1$, the constant $\beta \geq \max \left\{\alpha / p^{-}, 1\right\}$, and

$$
\left|g^{i}(x)\right| \leq c \rho^{\beta}(x)
$$

then for any $t \in[0, T)$, there holds

$$
\begin{aligned}
& \int_{\Omega}[u(x, t)-v(x, t)]^{2} d x \\
& \quad \leq c \int_{\Omega}[u(x, 0)-v(x, 0)]^{2} d x .
\end{aligned}
$$

Last but not least, no matter whether $\alpha<p^{-}-1$ or not, the uniqueness of the weak solutions is always true. Actually, similar as [19], we can prove the following theorem.

Theorem 4. If $b_{i}(s)$ is a Lipschitz function, $\alpha>0, p^{-}>1$, then the solution of (6) with the initial value (2) is unique.

However, for the simplicity of the paper, we will not give the details of the proof of Theorem 4 in what follows.

The rest of the paper is arranged as follows. In Section 2, Theorem 2 is proved. In Section 3, Theorem 3 is proved. In the last section, we give an explanation of the partial boundary value condition (8), and some conclusions similar to Theorem 2 are obtained without condition (14). 


\section{The Stability of Solutions When $\alpha<p^{-}-1$}

Lemma 5 (see [9]). (i) Let $p(x)$ and $q(x)$ be real functions with $1 / p(x)+1 / q(x)=1$ and $p(x)>1$. Then, for any $u \in L^{p(x)}(\Omega)$ and $v \in L^{q(x)}(\Omega)$, we have

$$
\left|\int_{\Omega} u v d x\right| \leq 2\|u\|_{L^{p(x)}(\Omega)}\|v\|_{L^{q(x)}(\Omega)}
$$

(ii)

$$
\begin{aligned}
& \text { If }\|u\|_{L^{p(x)}(\Omega)}=1, \\
& \text { then } \int_{\Omega}|u|^{p^{(x)}} d x=1, \\
& \text { If } \quad\|u\|_{L^{p(x)}(\Omega)}>1, \\
& \text { then }|u|_{L^{p(x)}}^{p^{-}} \leq \int_{\Omega}|u|^{p(x)} d x \leq|u|_{L^{p(x)}}^{p^{+}}, \\
& \text {If } \quad\|u\|_{L^{p(x)}(\Omega)}<1, \\
& \text { then }|u|_{L^{p(x)}}^{p^{+}} \leq \int_{\Omega}|u|^{p(x)} d x \leq|u|_{L^{p(x)}}^{p^{-}} .
\end{aligned}
$$

Proof of Theorem 2. For a small positive constant $\lambda>0$, let

$$
\phi(x)= \begin{cases}1, & \text { if } x \in \Omega_{\lambda}, \\ \frac{\rho}{\lambda}, & \text { if } x \in \Omega \backslash \Omega_{\lambda},\end{cases}
$$

where

$$
\Omega_{\lambda}=\{x \in \Omega: \rho(x)=\operatorname{dist}(x, \partial \Omega)>\lambda\} .
$$

Then

$$
\nabla \phi=\frac{1}{\lambda} \nabla \rho, \quad x \in \Omega \backslash \Omega_{\lambda}
$$

For small $\eta>0$, let

$$
\begin{aligned}
& S_{\eta}(s)=\int_{0}^{s} h_{\eta}(\tau) d \tau, \\
& h_{\eta}(s)=\frac{2}{\eta}\left(1-\frac{|s|}{\eta}\right)_{+} .
\end{aligned}
$$

Obviously, $h_{\eta}(s) \in C(\mathbb{R})$ and

$$
\begin{aligned}
h_{\eta}(s) & \geq 0, \\
\left|s h_{\eta}(s)\right| & \leq 1, \\
\left|S_{\eta}(s)\right| & \leq 1, \\
\lim _{\eta \rightarrow 0} S_{\eta}(s) & =\operatorname{sgn} s, \\
\lim _{\eta \rightarrow 0} s S_{\eta}^{\prime}(s) & =0 .
\end{aligned}
$$

If $u$ and $v$ are two weak solutions of (6) with the same partial homogeneous boundary value $(13)$ and $S_{\eta}(\phi(u-v))$ is chosen to be the test function, then

$$
\begin{aligned}
& \int_{\Omega} S_{\eta}(\phi(u-v)) \frac{\partial(u-v)}{\partial t} d x \\
& +\int_{\Omega} \rho^{\alpha}\left(|\nabla u|^{p(x)-2} \nabla u-|\nabla v|^{p(x)-2} \nabla v\right) \\
& \cdot \phi \nabla(u-v) S_{\eta}^{\prime}(\phi(u-v)) d x \\
& +\int_{\Omega} \rho^{\alpha}\left(|\nabla u|^{p(x)-2} \nabla u-|\nabla v|^{p(x)-2} \nabla v\right) \\
& \cdot \nabla \phi(u-v) S_{\eta}^{\prime}(\phi(u-v)) d x+\int_{\Omega} g_{x_{i}}^{i}(x) \\
& \cdot(b(u)-b(v)) S_{\eta}(\phi(u-v)) d x+\int_{\Omega} g^{i}(x) \\
& \cdot(b(u)-b(v))(u-v)_{x_{i}} S_{\eta}^{\prime}(\phi(u-v)) \phi d x \\
& +\int_{\Omega} g^{i}(x)(b(u)-b(v)) \phi_{x_{i}}(u-v) \\
& +S_{\eta}^{\prime}(\phi(u-v)) d x=0 .
\end{aligned}
$$

Thus

$$
\lim _{\eta \rightarrow 0} \lim _{\lambda \rightarrow 0} \int_{\Omega} S_{\eta}(\phi(u-v)) \frac{\partial(u-v)}{\partial t} d x=\frac{d}{d t}
$$

$$
\int_{\Omega}|u-v| d x
$$

$$
\begin{gathered}
\int_{\Omega} \rho^{\alpha}\left(|\nabla u|^{p(x)-2} \nabla u-|\nabla v|^{p(x)-2} \nabla v\right) \\
\cdot \phi \nabla(u-v) S_{\eta}^{\prime}(\phi(u-v)) d x \geq 0 .
\end{gathered}
$$

Obviously, we have

$$
\begin{aligned}
& \left|(u-v) S_{\eta}^{\prime}(\phi(u-v))\right|=\left|\phi(u-v) S_{\eta}^{\prime}(\phi(u-v))\right| \frac{1}{\phi} \\
& \quad \leq \frac{c}{\phi}
\end{aligned}
$$

$$
\frac{|\nabla \phi|}{\phi} \leq \frac{c}{\lambda}
$$

Using the Young inequality, we have

$$
\begin{gathered}
\mid \int_{\Omega} \rho^{\alpha}\left(|\nabla u|^{p(x)-2} \nabla u-|\nabla v|^{p(x)-2} \nabla v\right) \\
\cdot \nabla \phi(u-v) S_{\eta}^{\prime}(\phi(u-v)) d x \mid
\end{gathered}
$$




$$
\begin{aligned}
& \leq \int_{\Omega \backslash \Omega_{\lambda}} \rho^{\alpha}\left(|\nabla u|^{p(x)-1}+|\nabla v|^{p(x)-1}\right) \\
& \cdot \frac{|\nabla \phi|}{\phi}|\phi(u-v)| S_{\eta}^{\prime}(\phi(u-v)) d x \\
& \leq c \int_{\Omega \backslash \Omega_{\lambda}} \frac{1}{\lambda} \rho^{\alpha}\left(|\nabla u|^{p(x)-1}+|\nabla v|^{p(x)-1}\right)|\phi(u-v)| \\
& \cdot S_{\eta}^{\prime}(\phi(u-v)) d x \leq \frac{c}{\lambda} \\
& \cdot \int_{\Omega \backslash \Omega_{\lambda}} \rho^{\alpha-\alpha /(p(x)-1)} \rho^{\alpha /(p(x)-1)}\left(|\nabla u|^{p(x)-1}\right. \\
& \left.+|\nabla v|^{p(x)-1}\right) d x \\
& \leq c \int_{\Omega \backslash \Omega_{\lambda}}\left[\rho^{\alpha}\left(|\nabla u|^{p(x)}+|\nabla v|^{p(x)}\right)\right. \\
& \left.+\frac{1}{\lambda p(x)} \rho^{p(x)(\alpha-\alpha /(p(x)-1))}\right] d x,
\end{aligned}
$$

which goes to 0 as $\lambda \rightarrow 0$, due to the assumption that $p^{-}-1>$ $\alpha \geq\left(p^{-}-1\right) /\left(p^{+}-2\right)$ implies that

$$
\frac{1}{\lambda^{p(x)}} \rho^{p(x)(\alpha-\alpha /(p(x)-1))} \leq \lambda^{[\alpha-1-\alpha /(p(x)-1)] p(x)} \longrightarrow 0,
$$

while

$$
\begin{aligned}
& \mid \int_{\Omega} g^{i}(x)(b(u)-b(v)) S_{\eta}^{\prime}(\phi(u-v))(u-v) \\
& \cdot \phi_{x_{i}}(x) d x\left|\leq c \int_{\Omega \backslash \Omega_{\lambda}}\right| b(u)-b(v) \mid \frac{\left|g^{i}(x)\right|}{\lambda} d x .
\end{aligned}
$$

Since $b(s)$ is a Lipschitz function, $|b(u)-b(v)| \leq c \mid u-$ $v \mid$. According to the definition of the trace, by the partial boundary value condition (7),

$$
\begin{aligned}
& u(x, t)=v(x, t)=0, \\
& x \in \Sigma_{1}=\left\{x \in \partial \Omega: g^{i}(x) \neq 0\right\}, \\
& g^{i}(x)=0, \quad x \in \Sigma_{2}=\left\{x \in \partial \Omega: g^{i}(x)=0\right\},
\end{aligned}
$$

we have

$$
\begin{gathered}
\lim _{\lambda \rightarrow 0} \mid \int_{\Omega} g^{i}(x)(b(u)-b(v)) S_{\eta}^{\prime}(\phi(u-v))(u-v) \\
\cdot \phi_{x_{i}}(x) d x\left|\leq c \int_{\partial \Omega}\right| g^{i}(x)|| u-v \mid d \Sigma \\
\quad=c \int_{\Sigma_{1} \cup \Sigma_{2}}\left|g^{i}(x)\right||u-v| d \Sigma=0 .
\end{gathered}
$$

Moreover, as in [17], we can prove that

$$
\begin{aligned}
& \lim _{\eta \rightarrow 0} \lim _{\lambda \rightarrow 0} \int_{\Omega} g^{i}(x)(b(u)-b(v)) S_{\eta}^{\prime}(\phi(u-v))(u-v)_{x_{i}} \\
& \cdot \phi(x) d x=0 .
\end{aligned}
$$

The details of the proof of (33) are omitted here.
Once again,

$$
\begin{aligned}
& \lim _{\eta \rightarrow 0} \lim _{\lambda \rightarrow 0}\left|\int_{\Omega} g_{x_{i}}^{i}(x)(b(u)-b(v)) S_{\eta}(\phi(u-v)) d x\right| \\
& \quad \leq c \int_{\Omega}|u-v| d x .
\end{aligned}
$$

Now, after letting $\lambda \rightarrow 0$, let $\eta \rightarrow 0$ in (25). Then, by (26), (28), (32), (33), and (34), we have

$$
\frac{d}{d t} \int_{\Omega}|u-v| d x \leqslant c \int_{\Omega}|u-v| d x
$$

and by the Gronwall inequality, we have

$$
\begin{array}{r}
\int_{\Omega}|u(x, t)-v(x, t)| d x \leqslant c \int_{\Omega}\left|u_{0}(x)-v_{0}(x)\right| d x \\
\forall t \in[0, T) .
\end{array}
$$

Theorem 2 is proved.

\section{The Stability of Solutions without the Boundary Value Condition}

As we have said in the introduction, when $\alpha \geq p^{-}-1$, since the weak solutions of (6) generally lack the regularity, we cannot define the trace on the boundary. Thus, we cannot use the boundary value condition to research the stability or the uniqueness of the weak solution. In order to overcome this difficulty, we introduce another kind of the weak solutions as follows.

Definition 6. A function $u(x, t)$ is said to be a weak solution of (6) with the initial value (2), if $u$ satisfies

$$
\begin{array}{r}
u \in L^{\infty}\left(Q_{T}\right), \\
\frac{\partial u}{\partial t} \in L^{2}\left(Q_{T}\right), \\
\rho^{\alpha}|\nabla u|^{p(x)} \in L^{1}\left(Q_{T}\right),
\end{array}
$$

and for any function $\varphi_{1} \in L^{1}\left(0, T ; C_{0}^{1}(\Omega)\right), \varphi_{2} \in L^{\infty}\left(Q_{T}\right)$ such that for any given $t \in[0, T), \varphi_{2}(x, \cdot) \in W_{\mathrm{loc}}^{1, p(x)}(\Omega)$,

$$
\begin{aligned}
& \iint_{Q_{T}}\left[\frac{\partial u}{\partial t}\left(\varphi_{1} \varphi_{2}\right)+\rho^{\alpha}|\nabla u|^{p(x)-2} \nabla u \cdot \nabla\left(\varphi_{1} \varphi_{2}\right)\right. \\
& \quad+g^{i}(x) b(u)\left(\varphi_{1} \varphi_{2}\right)_{x_{i}} \\
& \left.\quad+b(u) g_{x_{i}}^{i}\left(\varphi_{1} \varphi_{2}\right)\right] d x d t=0,
\end{aligned}
$$

and the initial value (2) is satisfied in the sense of

$$
\lim _{t \rightarrow 0} \int_{\Omega}\left|u(x, t)-u_{0}(x)\right| d x=0
$$


We first introduced this kind of the weak solutions in our previous paper [19], in which the following equation was studied:

$$
\begin{aligned}
\frac{\partial u}{\partial t} & -\operatorname{div}\left(a(x)|\nabla u|^{p-2} \nabla u\right)-b^{i}(x) D_{i} u+c(x, t) u \\
& =f(x, t), \quad(x, t) \in \Omega \times(0, T),
\end{aligned}
$$

where $0 \leq a(x) \in C^{1}(\bar{\Omega})$ with $\left.a(x)\right|_{x \in \partial \Omega}=0$. It is not difficult to prove the existence of the weak solution in the sense of Definition 6.

Proof of Theorem 3. For any fixed $s, \tau \in[0, T)$, after an approximate procedure, we may choose $\chi_{[\tau, s]}(u-v) \phi$ as a test function in equality (38), where $\chi_{[\tau, s]}$ is the characteristic function on $[\tau, s]$ and $\phi(x)$ is defined as (20). Thus we have

$$
\begin{aligned}
& \iint_{Q_{\tau s}}(u-v) \phi \frac{\partial(u-v)}{\partial t} d x d t \\
& \quad=-\iint_{Q_{s}} \rho^{\alpha}\left(|\nabla u|^{p(x)-2} \nabla u-|\nabla v|^{p(x)-2} \nabla v\right) \\
& \cdot \nabla[(u-v) \phi] d x d t \\
& -\iint_{Q_{\tau s}} g^{i}(x)[b(u)-b(v)][(u-v) \phi]_{x_{i}} d x d t \\
& -\iint_{Q_{\tau s}}[b(u)-b(v)] g_{x_{i}}^{i} \phi(u-v) d x d t,
\end{aligned}
$$

where $Q_{\tau s}=\Omega \times[\tau, s]$.

We can rewrite (41) as follows:

$$
\begin{aligned}
& \int_{\Omega}[u(x, s)-v(x, s)]^{2} \phi d x=\int_{\Omega}[u(x, 0)-v(x, 0)]^{2} \\
& \cdot \phi d x \\
& -2 \iint_{Q_{\tau s}} \phi \rho^{\alpha}\left(|\nabla u|^{p(x)-2} \nabla u-|\nabla v|^{p(x)-2} \nabla v\right) \\
& \cdot \nabla(u-v) d x d t-2 \iint_{Q_{\tau s}}(u-v) \\
& \cdot \rho^{\alpha}\left(|\nabla u|^{p(x)-2} \nabla u-|\nabla v|^{p(x)-2} \nabla v\right) \nabla \phi d x d t \\
& -2 \iint_{Q_{\tau s}} g^{i}(x)[b(u)-b(v)] \\
& \cdot[(u-v) \phi]_{x_{i}} d x d t-2 \iint_{Q_{\tau s}}[b(u)-b(v)] \\
& \cdot g_{x_{i}}^{i} \phi(u-v) d x d t \\
& \leq \int_{\Omega}[u(x, 0)-v(x, 0)]^{2} \phi d x-2 \iint_{Q_{\tau s}}(u-v) \\
& \cdot \rho^{\alpha}\left(|\nabla u|^{p(x)-2} \nabla u-|\nabla v|^{p(x)-2} \nabla v\right) \nabla \phi d x d t
\end{aligned}
$$

$$
\begin{aligned}
& -2 \iint_{Q_{\tau s}} g^{i}(x)[b(u)-b(v)] \\
& \cdot[(u-v) \phi]_{x_{i}} d x d t-2 \iint_{Q_{\tau s}}[b(u)-b(v)] \\
& \cdot g_{x_{i}}^{i} \phi(u-v) d x d t .
\end{aligned}
$$

In the first place, since

$$
\begin{aligned}
& \mid-2 \iint_{Q_{\tau s}}(u-v) \rho^{\alpha}\left(|\nabla u|^{p(x)-2} \nabla u-|\nabla v|^{p(x)-2} \nabla v\right) \\
& \cdot \nabla \phi d x d t\left|\leq 2 \iint_{Q_{\tau s}}\right| u-v \mid \rho^{\alpha}\left(|\nabla u|^{p(x)-1}\right. \\
& \left.+|\nabla v|^{p(x)-1}\right)|\nabla \phi| d x d t \\
& \leq c \int_{\tau}^{s} \int_{\Omega \mid \Omega_{\lambda}}\left[\frac{p(x)-1}{p(x)} \rho^{\alpha}\left(|\nabla u|^{p(x)}+|\nabla v|^{p(x)}\right)\right. \\
& \left.+\frac{1}{p(x)} \rho^{\alpha}|\nabla \phi|^{p(x)}\right] d x d t \\
& \quad \leq c \int_{\tau}^{s} \int_{\Omega \mid \Omega_{\lambda}}\left[\frac{p(x)-1}{p(x)} \rho^{\alpha}\left(|\nabla u|^{p(x)}+|\nabla v|^{p(x)}\right)\right. \\
& \left.\quad+\frac{1}{p(x)} \lambda^{\alpha-p(x)}\right] d x d t,
\end{aligned}
$$

by $\alpha>p^{+}-1$ leading to $\alpha-p(x)>-1$, (43) yields

$$
\begin{aligned}
& \lim _{\lambda \rightarrow 0} \mid-2 \iint_{Q_{\tau s}}(u-v) \\
& \cdot \rho^{\alpha}\left(|\nabla u|^{p(x)-2} \nabla u-|\nabla v|^{p(x)-2} \nabla v\right) \nabla \phi d x d t \mid=0 .
\end{aligned}
$$

In the second place, since $\left|\rho_{x_{i}}\right| \leq|\nabla \rho|=1$, by (16), $\left|g^{i}(x)\right| \leq c \rho^{\beta}(x)$, then

$$
\begin{aligned}
& \lim _{\lambda \rightarrow 0}\left|\iint_{Q_{\tau s}} g^{i}(x)[b(u)-b(v)](u-v) \phi_{x_{i}} d x d t\right| \\
& =\lim _{\lambda \rightarrow 0}\left|\int_{\tau}^{s} \int_{\Omega \backslash \Omega_{\lambda}} g^{i}(x)[b(u)-b(v)](u-v) \phi_{x_{i}} d x d t\right| \\
& \leq c \lim _{\lambda \rightarrow 0} \int_{\tau}^{s} \int_{\Omega \backslash \Omega_{\lambda}}|u-v|^{2} d x d t=0 .
\end{aligned}
$$

By $u, v \in L^{\infty}\left(Q_{T}\right)$ and the condition (16), we have 


$$
\begin{aligned}
& \left|\iint_{Q_{\tau s}} g^{i}(x)[b(u)-b(v)](u-v)_{x_{i}} \phi d x d t\right| \\
& \quad \leq \sum_{i=1}^{N}\left(\int_{\tau}^{s} \int_{\Omega}\left|g^{i}(x)\right|^{p(x) /(p(x)-1)} \rho^{-\alpha /(p(x)-1)}|b(u)-b(v)|^{p(x) /(p(x)-1)} d x d t\right)^{1 / q_{1}} \\
& \quad \cdot\left(\int_{\tau}^{s} \int_{\Omega} \rho^{\alpha}\left(|\nabla u|^{p(x)}+|\nabla v|^{p(x)}\right) d x d t\right)^{1 / p_{1}} \leq c \sum_{i=1}^{N}\left(\int_{\tau}^{s} \int_{\Omega} \rho^{(\beta p(x) /(p(x)-1)-\alpha /(p(x)-1))}|u-v|^{p(x) /(p(x)-1)} d x d t\right)^{1 / q_{1}} \\
& \quad \leq c \sum_{i=1}^{N}\left(\int_{\tau}^{s} \int_{\Omega}|u-v|^{p(x) /(p(x)-1)} d x d t\right)^{1 / q_{1}}
\end{aligned}
$$

by which $\beta \geq \alpha / p^{-}$. Here $p_{1}=p^{+}$or $p^{-}$and $q_{1}=$ $\max \{p(x) /(p(x)-1)\}$ or $\min \{p(x) /(p(x)-1)\}$ according to (iii) of Lemma 5.

We denote that

$$
\begin{aligned}
& \Omega_{1}=\left\{x \in \Omega: \frac{p(x)}{p(x)-1} \geq 2\right\}, \\
& \Omega_{2}=\left\{x \in \Omega: \frac{p(x)}{p(x)-1}<2\right\},
\end{aligned}
$$

and then

$$
\begin{aligned}
& \int_{\Omega_{1}}|u-v|^{p(x) /(p(x)-1)} d x \leq c \int_{\Omega_{1}}|u-v|^{2} d x, \\
& \int_{\Omega_{2}}|u-v|^{p(x) /(p(x)-1)} d x \leq c\left(\int_{\Omega_{1}}|u-v|^{2} d x\right)^{1 / q_{2}},
\end{aligned}
$$

where $q_{2}=\max \{2(p(x)-1) / p(x)\}$ or $\min \{2(p(x)-1) / p(x)\}$ according to (iii) of Lemma 5 .

Combining (48) with (46), we have

$$
\begin{aligned}
& \left|\iint_{Q_{\tau s}} g^{i}(x)[b(u)-b(v)](u-v)_{x_{i}} \phi d x d t\right| \\
& \quad \leq c\left(\int_{\tau}^{s} \int_{\Omega}|u-v|^{2} d x d t\right)^{l},
\end{aligned}
$$

where $l<1$.

Once more,

$$
\begin{aligned}
& \left|\iint_{Q_{\tau s}}[b(u)-b(v)] \phi(u-v) g_{x_{i}}^{i} d x d t\right| \\
& \quad \leq c \int_{\tau}^{s} \int_{\Omega}|u-v|^{2} d x d t .
\end{aligned}
$$

Let $\lambda \rightarrow 0$ in (42). By (44), (45), (49), and (50), we have

$$
\begin{aligned}
& \int_{\Omega}[u(x, s)-v(x, s)]^{2} d x \\
& \leq \int_{\Omega}[u(x, 0)-v(x, 0)]^{2} d x \\
& \quad+c \int_{\tau}^{s} \int_{\Omega}|u-v|^{2} d x d t \\
& \quad+c\left(\int_{\tau}^{s} \int_{\Omega}|u-v|^{2} d x d t\right)^{l} .
\end{aligned}
$$

Let $\kappa(s)=\int_{\Omega}|u(x, s)-v(x, s)|^{2} d x$. Without loss of the generality, we may assume that there exist $\tau \in[0, T)$ and $\kappa(\tau)>0$. Then for any $s>\tau, \int_{\tau}^{s} k(t) d t>0$. If we denote that

$$
\begin{aligned}
\tau_{0} & =\sup \{t \in[\tau, s], \kappa(t)>0\}, \\
\int_{\tau}^{\tau_{0}} k(t) d t & =c_{1},
\end{aligned}
$$

then $\tau<\tau_{0} \leq s$ and

$$
\int_{\tau}^{s} k(t) d t \geq \int_{\tau}^{\tau_{0}} k(t) d t=c_{1} .
$$

By $u, v \in L^{\infty}\left(Q_{T}\right)$, there exists a constant $C>0$ such that

$$
\begin{aligned}
\frac{c\left(\int_{\tau}^{s} k(t) d t\right)^{l}}{\int_{\tau}^{s} k(t) d t} & \leq \frac{c\left(\int_{\tau}^{s} k(t) d t\right)^{l}}{c_{1}} \leq C \\
& =C\left(c, c_{1}, T, q\right) .
\end{aligned}
$$

By (51) and (54), we have

$$
\kappa(s)-\kappa(\tau) \leq(C+c) \int_{\tau}^{s} k(t) d t,
$$

and using the Gronwall inequality, we have

$$
\begin{aligned}
& \int_{\Omega}|u(x, s)-v(x, s)|^{2} d x \\
& \quad \leq c \int_{\Omega}|u(x, \tau)-v(x, \tau)|^{2} d x,
\end{aligned}
$$


where $c$ depends on $C$. Thus, we have

$$
\int_{\Omega}|u(x, s)-v(x, s)|^{2} d x \leq c \int_{\Omega}\left|u_{0}(x)-v_{0}(x)\right| d x .
$$

The proof is complete.

\section{The Partial Boundary Value Condition}

Consider the linear degenerate equation

$$
\frac{\partial u}{\partial t}-\operatorname{div}(a(x) \nabla u)-\sum_{i=1}^{N} f^{i}(x) D_{i} u=0,
$$

which is a particular case of (6) (where $p=2, g^{i}=f^{i}, b(s)=$ $s)$. Rewrite it as

$$
\frac{\partial u}{\partial t}-a(x) \Delta u-\sum_{i=1}^{N}\left(a_{x_{i}}(x)+f^{i}(x)\right) D_{i} u=0 .
$$

According to the Fichera-Oleinik theory $[20,21]$, besides the initial value condition (2), since $\left.a(x)\right|_{\partial \Omega}=0$, the partial boundary, where we should impose the boundary value condition, is

$$
\Sigma_{1}=\left\{x \in \partial \Omega: \sum_{i=1}^{N} f^{i}(x) n_{i}(x)<0\right\},
$$

where $\vec{n}=\left\{n_{i}\right\}$ is the inner normal vector of $\partial \Omega$.

By reviewing the formula of (8), One can see that

$$
\Sigma_{1} \subseteq \Sigma_{p} \subseteq \partial \Omega
$$

By this token, condition (13) in Theorem 2 is reasonable.

\section{Conflicts of Interest}

The authors declare that there are no conflicts of interest regarding the publication of this paper.

\section{Acknowledgments}

The paper is supported by NSF of Fujian Province (no. 2015J01592), China.

\section{References}

[1] M. Ružicka, Electrorheological Fluids: Modeling and Mathematical Theory, Lecture Notes in Math, vol. 1748, Springer, Berlin, Germany, 2000.

[2] E. Acerbi and G. Mingione, "Regularity results for stationary electro-rheological fluids," Archive for Rational Mechanics and Analysis, vol. 164, no. 3, pp. 213-259, 2002.

[3] S. Antontsev and S. Shmarev, "Anisotropic parabolic equations with variable nonlinearity," Publicacions Matemàtiques, vol. 53, no. 2, pp. 355-399, 2009.

[4] S. Lian, W. Gao, H. Yuan, and C. Cao, "Existence of solutions to an initial Dirichlet problem of evolutional $\mathrm{p}(\mathrm{x})$-Laplace equations," Annales de l'Institut Henri Poincare (C) Non Linear Analysis, vol. 29, no. 3, pp. 377-399, 2012.
[5] C. Zhang, S. Zhou, and X. Xue, "Global gradient estimates for the parabolic $\mathrm{p}(\mathrm{x}, \mathrm{t})$-Laplacian equation," Nonlinear Analysis: Theory, Methods \& Applications, vol. 105, pp. 86-101, 2014.

[6] F. Yao, "Hölder regularity for the general parabolic $p(x, t)$ Laplacian equations," Nonlinear Differential Equations and Applications, vol. 22, no. 1, pp. 105-119, 2015.

[7] J. Yin and C. Wang, "Evolutionary weighted $p$-Laplacian with boundary degeneracy," Journal of Differential Equations, vol. 237, no. 2, pp. 421-445, 2007.

[8] J. Yin and C. Wang, "Properties of the boundary flux of a singular diffusion process," Chinese Annals of Mathematics, vol. 25B, no. 2, pp. 175-182, 2004.

[9] V. V. Zhikov, "On the density of smooth functions in SobolevOrlicz spaces," Journal of Mathematical Sciences, vol. 132, no. 3, pp. 285-294, 2004.

[10] X. L. Fan and D. Zhao, "On the spaces $L^{p(x)}(\Omega)$ and $W^{m, p(x)}(\Omega)$," Journal of Mathematical Analysis and Applications, vol. 263, no. 2, pp. 424-446, 2001.

[11] O. Kovácik and J. Rákosnk, "On spaces $L^{p(x)}$ and $W^{1, p(x), "}$ Czechoslovak Mathematical Journal, vol. 41, no. 116, pp. 592-618, 1991.

[12] L. Diening, P. Harjulehto, P. Hästö, and M. Ruzicka, Lebesgue and Sobolev Spaces with Variable Exponents, Lecture Notes in Mathematics, Springer, Berlin, Germany, 2017.

[13] M. A. Ragusa and A. Tachikawa, "On interior regularity of minimizers of $\mathrm{p}(\mathrm{x})$-energy functionals," Nonlinear Analysis, vol. 93, pp. 162-167, 2013.

[14] D. Cruz-Uribe, A. Fiorenza, and O. M. Guzmán, "Embeddings between grand, small, and variable Lebesgue spaces," Mathematical Notes, vol. 102, pp. 677-686, 2017.

[15] D. Cruz-Uribe, A. Fiorenza, and C. J. Neugebauer, "The maximal function on variable $L^{p(x)}$ spaces," Annales Academiae Scientiarum Fennicae Mathematica, vol. 28, no. 1, pp. 223-238, 2003.

[16] L. Diening, "Maximal function on generalized Lebesgue spaces $L^{p}(\cdot)$, , Mathematical Inequalities \& Applications, vol. 7, no. 2, pp. 339-351, 2004.

[17] H. Zhan, "The boundary value condition of an evolutionary $p(x)$-Laplacian equation," Boundary Value Problems, 2015:112, 24 pages, 2015.

[18] H. Zhan and J. Wen, "Evolutionary $p(x)$-Laplacian equation free from the limitation of the boundary value," Electronic Journal of Differential Equations, vol. 143, pp. 1-13, 2016.

[19] H. Zhan and B. Xu, "A new kind of weak solution of nonNewtonian fluid equation," Journal of Function Spaces, vol. 2017, Article ID 7916730, 8 pages, 2017.

[20] G. Fichera, "Sulle equazioni differenziali lineari ellitticoparaboliche del secondo ordine," Atti Accad. Naz. Lincei. Mem. Cl. Sci. Fis. Mat. Nat. Sez. I. VIII, ser. 5, pp. 3-30, 1956.

[21] O. A. Oleinik and E. V. Radkevic, Second Order Differential Equations with Nonnegative Characteristic Form, American Mathematical Society, Rhode Island, RI, USA and Plenum Press, New York, NY, USA, 1973. 


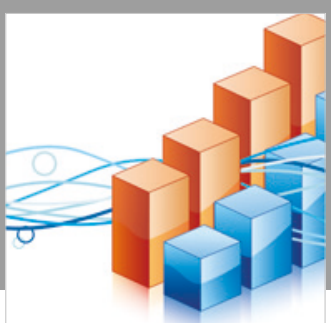

Advances in

Operations Research

\section{-n-m}
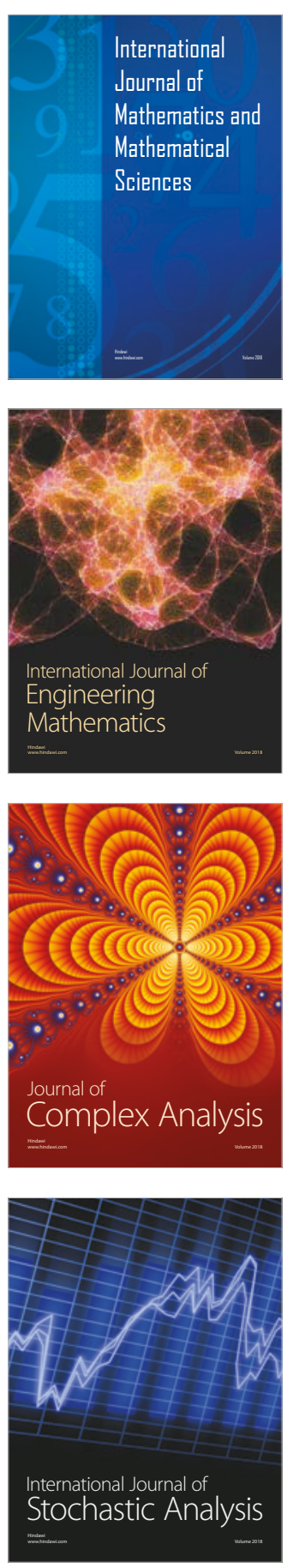
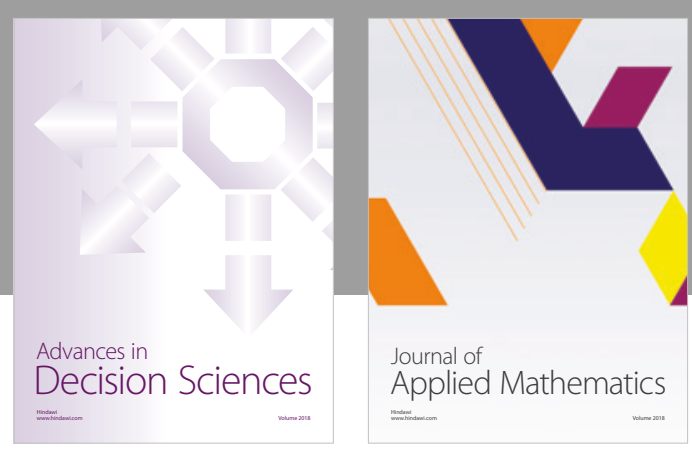

Journal of

Applied Mathematics
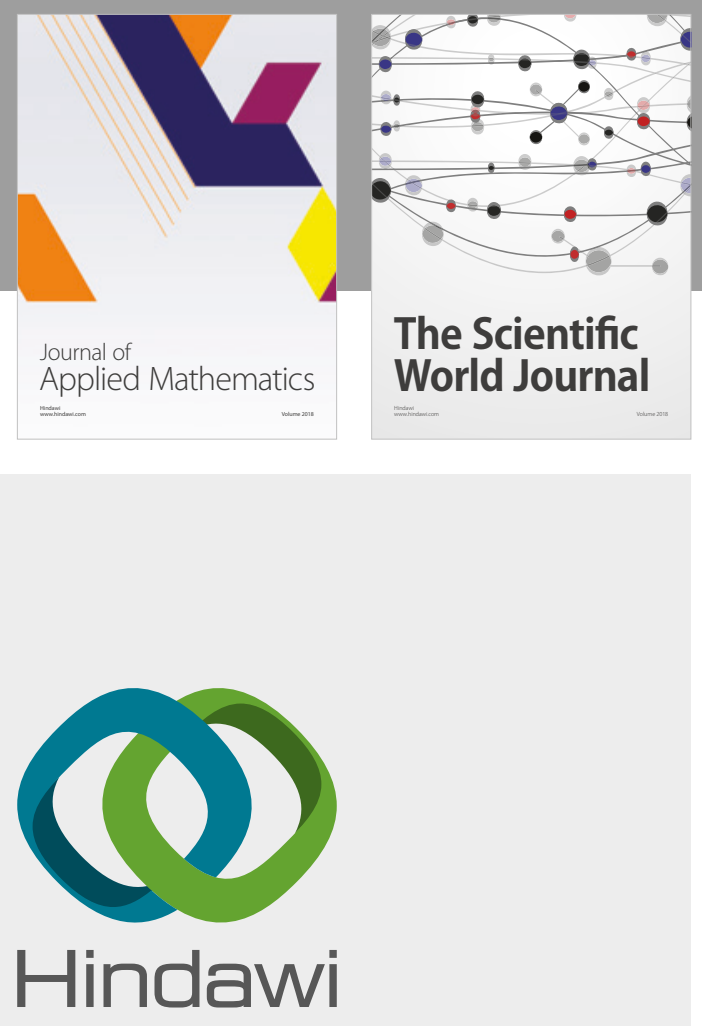

Submit your manuscripts at

www.hindawi.com

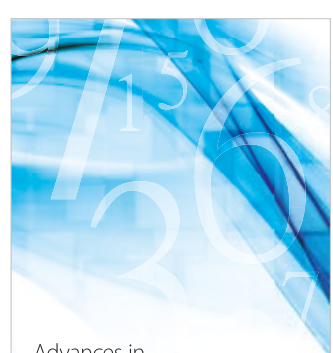

Advances in
Numerical Analysis
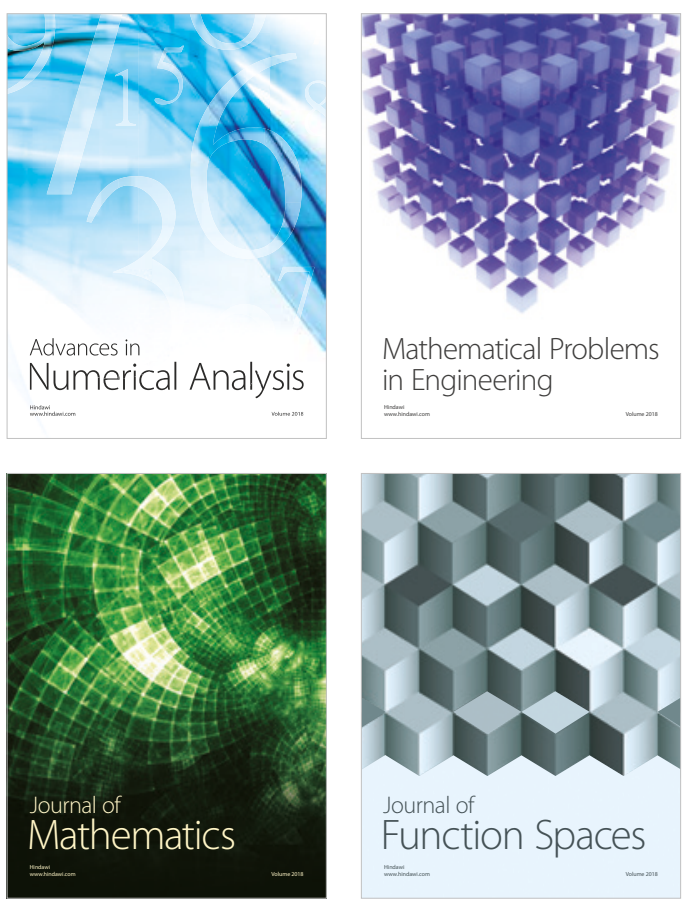

Mathematical Problems in Engineering

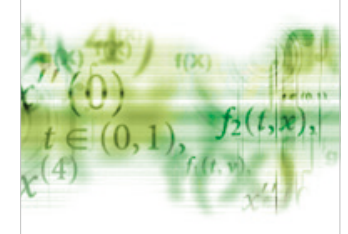

International Journal of

Differential Equations

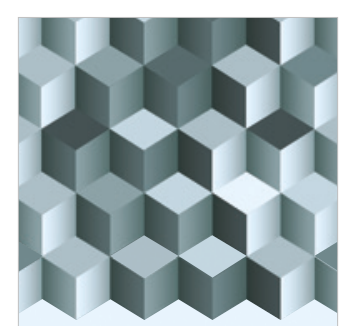

Journal of

Function Spaces

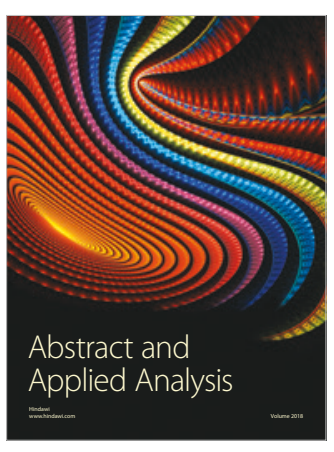

The Scientific

World Journal

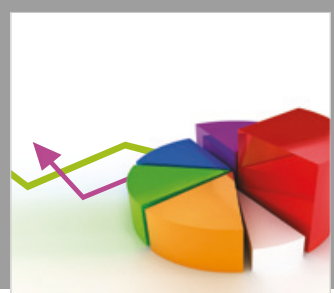

Journal of

Probability and Statistics
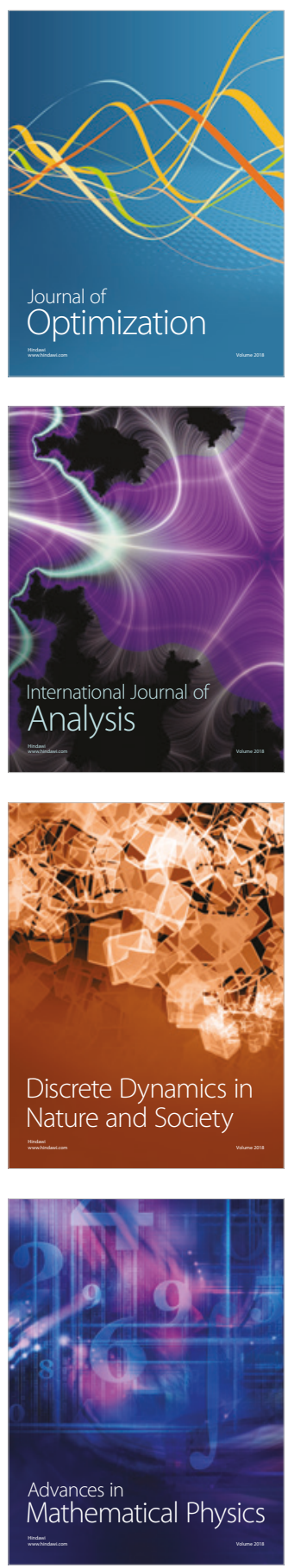